\title{
THE INTEGER CHEBYSHEV PROBLEM
}

\author{
PETER BORWEIN AND TAMÁS ERDÉLYI
}

\begin{abstract}
We are concerned with the problem of minimizing the supremum norm on an interval of a nonzero polynomial of degree at most $n$ with integer coefficients. This is an old and hard problem that cannot be exactly solved in any nontrivial cases.

We examine the case of the interval $[0,1]$ in most detail. Here we improve the known bounds a small but interesting amount. This allows us to garner further information about the structure of such minimal polynomials and their factors. This is primarily a (substantial) computational exercise.

We also examine some of the structure of such minimal "integer Chebyshev" polynomials, showing for example that on small intevals $[0, \delta]$ and for small degrees $d, x^{d}$ achieves the minimal norm. There is a natural conjecture, due to the Chudnovskys and others, as to what the "integer transfinite diameter" of $[0,1]$ should be. We show that this conjecture is false.

The problem is then related to a trace problem for totally positive algebraic integers due to Schur and Siegel. Several open problems are raised.
\end{abstract}

\section{INTRODUCTION}

The ubiquitous Chebyshev polynomial

$$
T_{n}(x):=\cos (n \arccos x)=\frac{1}{2}\left[\left(x+\sqrt{x^{2}-1}\right)^{n}+\left(x-\sqrt{x^{2}-1}\right)^{n}\right]
$$

is a polynomial of degree $n$ with integer coefficients and with lead coefficient $2^{n-1}$ that equioscillates $n+1$ times on the interval $[-1,1]$. For fairly simple reasons, based on this equioscillation, it follows that

$$
\min _{p_{n-1} \in \mathcal{P}_{n-1}}\left\|x^{n}-p_{n-1}\right\|_{[-1,1]}=\left\|2^{1-n} T_{n}\right\|_{[-1,1]}=\frac{1}{2^{n-1}} .
$$

Here and throughout, $\mathcal{P}_{n}$ denotes the set of all polynomials of degree at most $n$ with real coefficients, and $\|\cdot\|_{A}$ denotes the supremum norm on a set $A$. We denote by $\mathcal{Z}_{n}$ the set of all polynomials of degree at most $n$ with integer coefficients.

The polynomial

$$
p_{n}(x):=2\left(\frac{b-a}{4}\right)^{n} T_{n}\left(\frac{2 x-a-b}{b-a}\right)
$$

Received by the editor April 25, 1994 and, in revised form, September 5, 1994 and February 12, 1995.

1991 Mathematics Subject Classification. Primary 11J54, 11B83.

Key words and phrases. Transfinite diameter, integers, diophantine approximation, trace, Chebyshev, polynomial.

Research supported in part by NSERC of Canada. 
is now the monic polynomial of degree $n$ of smallest supremum norm on the interval $[a, b]$, and it satisfies

$$
\left\|p_{n}\right\|_{[a, b]}=2\left(\frac{b-a}{4}\right)^{n}
$$

The Chebyshev polynomials have a central role to play in minimization problems in the supremum norm as well as many other extremal problems. See, for example, $[6]$.

The analogous problem where the polynomials are restricted to have integer coefficients is very much harder. For a very nice discussion of this problem, see [15].

We define

$$
\Omega_{n}[a, b]:=\left(\inf _{0 \neq p \in \mathcal{Z}_{n}}\|p\|_{[a, b]}\right)^{1 / n}
$$

and let

$$
\Omega[a, b]:=\inf \left\{\Omega_{n}[a, b]: n=0,1, \ldots\right\}=\lim _{n \rightarrow \infty} \Omega_{n}[a, b]
$$

We call any polynomial $p \in \mathcal{Z}_{n}$ that achieves $\Omega_{n}[a, b]$ an $n$th integer Chebyshev polynomial on $[a, b]$. The above limit exists and equals the infimum because

$$
\Omega_{n}[a, b] \geq \min \left\{1,2^{1 / n} \frac{b-a}{4}\right\}
$$

as follows from the unrestricted case (1.3), and because

$$
\left(\Omega_{n+m}[a, b]\right)^{n+m} \leq\left(\Omega_{n}[a, b]\right)^{n}\left(\Omega_{m}[a, b)\right)^{m}
$$

since $\|r s\|_{[a, b]} \leq\|r\|_{[a, b]}\|s\|_{[a, b]}$. See, for example, [17, Chapter 3, §1, Problem 98].

We have the trivial inequality

$$
\Omega[a, b] \geq \min \left\{1, \frac{b-a}{4}\right\}
$$

We also have

$$
\Omega[a, b] \leq \Omega_{n}[a, b]
$$

for any particular $n$. Thus, good upper bounds can be achieved by computation (although the computation to any degree of accuracy is hard). The limit $\Omega[a, b]$ may be thought of as an integer version of the transfinite diameter.

Since $p=1$ is a candidate for achieving the infimum in (1.4) and since in (1.3), $2((b-a) / 4)^{n}>1$ if $b-a \geq 4$, we observe that on intervals $[a, b]$ of length greater than or equal to 4 we have that $\Omega_{n}[a, b]=\Omega[a, b]=1$. We will thus from now on restrict our attention to intervals of length at most 4 . 
Hilbert [12] showed that there exists an absolute constant $c$ so that

$$
\inf _{0 \neq p \in \mathcal{Z}_{n}}\|p\|_{L_{2}[a, b]} \leq c n^{1 / 2}\left(\frac{b-a}{4}\right)^{1 / 2}
$$

and Fekete [9] showed that

$$
\left(\Omega_{n}[a, b]\right)^{n} \leq 2^{1-2^{-n-1}}(n-1)\left(\frac{b-a}{4}\right)^{n / 2}
$$

For refinements of their inequalities, see Kashin [13].

From the above it follows that

$$
\frac{b-a}{4} \leq \Omega[a, b] \leq\left(\frac{b-a}{4}\right)^{1 / 2}
$$

Recall that $b-a \leq 4$.

There is a pretty argument due to Gelfond [see 10] to see that integer coefficients really are a restriction on $[0,1]$. If $0 \neq p_{n} \in \mathcal{Z}_{n}$, then for some integer $m \neq 0$,

$$
\left\|p_{n}\right\|_{[0,1]}^{2} \geq\left\|p_{n}\right\|_{L_{2}[0,1]}^{2}=\int_{0}^{1} p_{n}^{2}(x) d x=\frac{m}{\operatorname{LCM}(1,2, \ldots, 2 n+1)} \neq 0
$$

where LCM denotes the least common multiple. Now $\operatorname{LCM}(1,2, \ldots, n))^{1 / n} \sim e$, by the prime number theorem, and it follows that

$$
\Omega[0,1] \geq 1 / e
$$

This is not, however, the right lower bound.

The best previous bounds known on $[0,1]$ give

$$
\frac{1}{2.37684 \ldots} \leq \Omega[0,1] \leq \frac{1}{2.3541 \ldots} .
$$

See Aparicio [2,3,4,5], Amoroso [1], and the references therein. The upper bound is in Amoroso [1]. The lower bound is based on a method attributed to a number of people and variously rediscovered. (Aparicio [3] attributes it to Gorshkov.) It amounts to, in our context, showing that for every fixed $p_{n} \in \mathcal{Z}_{n}$ there exist infinitely many polynomials $q_{k} \in \mathcal{Z}_{n_{k}}$ with no common factors with $p_{n}$, with all their roots in $(0,1)$, and with leading coefficients $a_{n_{k}}$ satisfying

$$
a_{n_{k}}^{1 / n_{k}}<2.37684 \ldots
$$

(see $\S 3$ ). This argument rests on the following lemma, which is an easy consequence of the fundamental theorem of symmetric polynomials. See, for example, [8]. 
Lemma 1.1. Suppose $p_{n} \in \mathcal{Z}_{n}$ and suppose $q_{k}(z):=a_{k} z^{k}+\cdots+a_{0} \in \mathcal{Z}_{k}$ has all its roots in $[a, b]$. If $p_{n}$ and $q_{k}$ do not have common factors, then

$$
\left(\left\|p_{n}\right\|_{[a, b]}\right)^{1 / n} \geq\left|a_{k}\right|^{-1 / k} .
$$

Proof. Let $\beta_{1}, \beta_{2}, \ldots, \beta_{k}$ be the roots of $q_{k}$. Then

$$
\left|a_{k}\right|^{n} p_{n}\left(\beta_{1}\right) p_{n}\left(\beta_{2}\right) \cdots p_{n}\left(\beta_{k}\right)
$$

is a nonzero integer, and the result follows.

Aparicio also shows that if $[a, b]=[0,1]$, then any polynomial $p \in \mathcal{Z}_{n}$ for which the infimum in (1.4) is achieved, for sufficiently large $n$, has a factor of the form

$$
(x)^{\left\lfloor\lambda_{1} n\right\rfloor}(1-x)^{\left\lfloor\lambda_{1} n\right\rfloor}(2 x-1)^{\left\lfloor\lambda_{2} n\right\rfloor}\left(5 x^{2}-5 x+1\right)^{\left\lfloor\lambda_{3} n\right\rfloor},
$$

where $\lambda_{1} \geq 0.014, \lambda_{2} \geq 0.016$, and $\lambda_{3} \geq 0.0037$.

In $\S 2$ we improve the upper bounds of $\Omega[0,1]$ to

$$
\Omega[0,1] \leq \frac{1}{2.3605 \ldots}
$$

and use this to increase the number of factors that must divide an $n$th integer Chebyshev polynomial $T_{n}$ on $[0,1]$. The analysis, and in particular the approach to the computations on $\Omega[0,1]$, is the content of $\S 2$.

In $\S 3$ we improve, by different methods, the lower bound of the multiplicity of the zero of $T_{n}$ at 0 and 1 . We use this to show, in Theorem 3.4, that the natural lower bound of (1.9) is not the best possible lower bound. This disproves a conjecture of the Chudnovskys [8].

We also establish a lower bound for the multiplicity of the zero at 0 of the integer Chebyshev polynomial $T_{n, a}$ on $[a, 1]$. This is used to show that there exists a constant $\delta>0$ so that for every $a \in[-\delta, \delta]$ and sufficiently large $n$, the $n$th integer Chebyshev polynomials $T_{n, a}$ on $[0,1+a]$ are just the $n$th integer Chebyshev polynomials $T_{n, 0}$ on $[0,1]$. It follows that the function $\Omega(x):=\Omega[0, x]$ is constant on $[1-\delta, 1+\delta]$. This parallels various results of Amoroso [1]. In particular, Amoroso shows that there exists a constant $\delta>0$ so that for every $a \in[-\delta, \delta]$,

$$
\Omega\left[p_{1} / q_{1}, p_{2} / q_{2}\right]=\Omega\left[p_{1} / q_{1}-a, p_{2} / q_{2}+a\right]
$$

if $p_{1}, q_{1}, p_{2}$, and $q_{2}$ are positive integers with $p_{1} q_{2}-p_{2} q_{1}=1$. Our methods are quite distinct.

Analogues of the $[0,1]$ case are also established on intervals $[0,1 / m], m \in \mathbb{N}$. It follows, for instance, that the there exists an absolute constant $c>0$ so that $T_{n}(x)=x^{n}$ for every $n \leq c m$, where $T_{n}$ denotes an arbitrary $n$th integer Chebyshev polynomial on $[0,1 / m]$. We also show that

$$
\limsup _{m \in \mathbb{N}}\left((\Omega[0,1 / m])^{-1}-m\right)<2-\epsilon
$$

for some $\epsilon>0$.

In $\S 4$ we relate the integer Chebyshev problem on small intervals $[0,1 / m]$ to an old problem of Schur and Siegel on the trace of totally positive algebraic integers.

We conclude with a number of open problems. 


\section{Integer Chebyshev Polynomials on $[0,1]$}

We restrict our attention to the interval $[0,1]$. Though we observe in passing that

$$
(\Omega[-1,1])^{4}=(\Omega[0,1])^{2}=\Omega[0,1 / 4]
$$

as a consequence of the changes of variable $x \rightarrow x^{2}$ and $x \rightarrow x(1-x)$ and symmetry. (The dependence of the constant $\Omega[a, b]$ and the minimal polynomials on $[a, b]$ is interesting and is explored a little further in later sections.) Even computing lowdegree examples is complicated.

$$
\begin{array}{cccl}
\underline{n} & n \text {th integer Chebyshev polynomial on }[0,1] & & \underline{\Omega_{n}[0,1]} \\
\cline { 2 - 4 } 1 & x \text { or }(1-x) \text { or }(2 x-1) & 1 \\
2 & & x(1-x) & 1 / 2 \\
3 & x(1-x)(2 x-1) & 1 /(2.18 \ldots) \\
4 & x^{2}(1-x)^{2} \text { or } x(1-x)(2 x-1)^{2} & 1 / 2 \\
5 & x^{2}(1-x)^{2}(2 x-1) & 1 /(2.23 \ldots) \\
6 & & {[x(1-x)(2 x-1)]^{2}} & 1 /(2.18 \ldots)
\end{array}
$$

Note that we do not have uniqueness, though it is open as to whether we have uniqueness for $n$ sufficiently large. The arguments for the above table are of the following variety. Consider, for example, the case $n=5$. Let $T_{5} \in \mathcal{Z}_{5}$ be a 5 th integer Chebyshev polynomial on $[0,1]$. Then $T_{5}(0)$ and $T_{5}(1)$ are integers of modulus less than $1 /(2.23 \ldots)^{5}$, so both of them must be 0 . Using Markov's inequality, we obtain that

$$
\left\|T_{5}^{\prime}\right\|_{[0,1]} \leq 50\left\|T_{5}\right\|_{[0,1]} \leq \frac{50}{(2.23 \ldots)^{5}}<1 .
$$

Since $T_{5}^{\prime}(0)$ and $T_{5}^{\prime}(1)$ are integers of modulus less than 1 , both of them must be zero. Since $2^{5} T_{5}(1 / 2)$ is an integer of modulus at most $32 /(2.236 \ldots)^{5}<1$, it must also be zero. Thus we have

$$
x^{2}(1-x)^{2}(2 x-1) \text { divides } T_{5},
$$

and the result follows.

Let

$$
\begin{aligned}
p_{0}(x):= & \\
p_{1}(x):= & =x, \\
p_{2}(x):= & =2 x-1, \\
p_{3}(x):= & 5 x^{2}-5 x+1, \\
p_{4}(x):= & 13 x^{3}-19 x^{2}+8 x-1, \\
p_{5}(x):= & 13 x^{3}-20 x^{2}+9 x-1, \\
p_{6}(x):= & 29 x^{4}-58 x^{3}+40 x^{2}-11 x+1, \\
p_{7}(x):= & 31 x^{4}-61 x^{3}+41 x^{2}-11 x+1, \\
p_{8}(x):= & 31 x^{4}-63 x^{3}+44 x^{2}-12 x+1, \\
p_{9}(x):= & 941 x^{8}-3764 x^{7}+6349 x^{6}-5873 x^{5} \\
& +3243 x^{4}-1089 x^{3}+216 x^{2}-23 x+1 .
\end{aligned}
$$

We have 
Proposition 2.1. Let

$$
P_{210}:=p_{0}^{67} \cdot p_{1}^{67} \cdot p_{2}^{24} \cdot p_{3}^{9} \cdot p_{4} \cdot p_{5} \cdot p_{6}^{3} \cdot p_{7} \cdot p_{8} \cdot p_{9}
$$

then

$$
\left(\left\|P_{210}\right\|_{[0,1]}\right)^{1 / 210}=\frac{1}{2.3543 \ldots}
$$

and hence

$$
\Omega[0,1] \leq \frac{1}{2.3543 \ldots}
$$

Proof. This proof is obviously just a computational verification. It is the algorithm for finding $P_{210}$ which is of some interest. It is based on $L L L$ lattice basis reduction [14] in the following way.

a] Lattice basis reduction finds a short vector in a lattice. If we construct a lattice of the form

$$
p(z) \cdot \sum_{k=0}^{n} \alpha_{k} z^{k}=\sum_{k=0}^{m} \beta_{k} z^{k},
$$

where $p$ is a fixed polynomial and the set $\left\{\left(\alpha_{0}, \alpha_{1}, \ldots, \alpha_{n}\right)\right\}$ is a lattice, then the set $\left\{\left(\beta_{0}, \beta_{1}, \ldots, \beta_{m}\right)\right\}$ is also a lattice, and $L L L$ will return a short vector in the sense of $\sum_{k=0}^{m}\left|\beta_{k}\right|^{2}$ being relatively small. Observe that $\left(\sum_{k=0}^{m}\left|\beta_{k}\right|^{2}\right)^{1 / 2}$ is just the $L_{2}$ norm on the unit disk of the polynomial $\sum_{k=0}^{m} \beta_{k} z^{k}$. So $L L L$ lets us find polynomials of small $L_{2}$ norm (and hence small sup norm) on the disk, and we can do this while preserving divisibility by a fixed $p$.

b] Convert the problem from the interval $[\alpha, \beta]$ to the disk. This is easy. One first maps $[\alpha, \beta]$ to $[-2,2]$ by a linear change of variables. One then lets $x:=z+1 / z$. This maps a polynomial in $x$ on $[-2,2]$ to a polynomial in $z$ and $1 / z$ on the boundary of the unit disk.

c] Attack the problem incrementally by using a] and b]. That is, at the $k$ th stage find a polynomial $q_{k}$ of degree $k N$ divisible by $q_{k-1}$ of degree $(k-1) N$ using $L L L$ on a lattice of size $N+1$. This allows us to keep the size of $L L L$ fairly small and uses the fact that integer Chebyshev polynomials tend to have (of necessity) many repeated factors. We used $N=10$ in the actual computation and started with $q_{0}:=1$.

We can computationally refine the above proposition as follows:

Proposition 2.2. The following inequalities hold:

$$
\begin{gathered}
\Omega[0,1 / 4] \leq \frac{1}{5.5723 \ldots}, \\
\Omega[0,1] \leq \frac{1}{2.3605 \ldots} .
\end{gathered}
$$

Proof. We minimize

$$
\left\|S_{1}^{\alpha_{1}} S_{2}^{\alpha_{2}} \cdots S_{9}^{\alpha_{9}}\right\|_{[0,1 / 4]},
$$


where

$$
\begin{aligned}
& S_{1}(x):=x \\
& S_{2}(x):=4 x-1 \\
& S_{3}(x):=5 x-1 \\
& S_{4}(x):=29 x^{2}-11 x+1 \\
& S_{5}(x):=169 x^{3}-94 x^{2}+17 x-1, \\
& S_{6}(x):=961 x^{4}-712 x^{3}+194 x^{2}-23 x+1, \\
& S_{7}(x):=941 x^{4}-703 x^{3}+193 x^{2}-23 x+1, \\
& S_{8}(x):=969581 x^{8}-1441511 x^{7}+928579 x^{6}-338252 x^{5} \\
&+76143 x^{4}-10836 x^{3}+951 x^{2}-47 x+1, \\
& S_{9}(x):= 6 x-1,
\end{aligned}
$$

by using the simplex method on a large grid of points $\left\{x_{k}\right\}_{k=1}^{N}$ that includes all the extreme points of the above polynomials in $[0,1 / 4]$ to minimize the linear system

$$
\frac{1}{2} \sum_{i=1}^{9} \alpha_{i} \log S_{i}^{2}\left(x_{k}\right), \quad k=1,2, \ldots, N
$$

subject to

$$
\sum_{i=1}^{9} \alpha_{i}=1, \quad \alpha_{i} \geq 0, \quad i=1,2, \ldots, 9 .
$$

If we do this, we obtain that

$$
\left[\alpha_{1}, \alpha_{2}, \ldots, \alpha_{9}\right]
$$$$
:=[.719122, .13093, .083914, .02928, .01450, .005154, .01028, .003612, .003189]
$$

and can check that

$$
\left\|S_{1}^{\alpha_{1}} S_{2}^{\alpha_{2}} \cdots S_{9}^{\alpha_{9}}\right\| \leq \frac{1}{5.5724 \ldots} .
$$

Now raise the whole result to a power that makes each $\alpha_{i}$ integer $\left(10^{10}\right.$ works $)$ and the first inequality of the proposition follows. The second inequality follows from (2.1).

The choice of $S_{1}, S_{2}, \ldots, S_{7}$ comes about on transforming $p_{1}, p_{2}, \ldots, p_{9}$ from $[0,1]$ to $[0,1 / 4]$ by $x \rightarrow x(1-x)$. So, for example, $\left(p_{4} p_{5}\right)(x)=S_{5}(x(1-x))$. The remaining two polynomials $S_{8}$ and $S_{9}$ were chosen somewhat gratuitously (though $S_{9}$ is the next in a particular sequence of totally real polynomials with roots in $[0,1 / 4]$ and with small leading coefficient).

We could also have solved the above minimization problem using Remez's algorithm. 
Corollary 2.3. Let $k$ be a positive integer, and let $P_{210}$ be as in Proposition 2.3. Then $\left(P_{210}\right)^{k}$ divides all the $n$th integer Chebyshev polynomials on $[0,1]$ provided $n$ is sufficiently large.

Proof. Each $p_{i}, i=0,1, \ldots, 9$, is irreducible and satisfies

$$
p_{i}(x)=a_{k} x^{k}+a_{k-1} x^{k-1}+\cdots+a_{0}
$$

with

$$
\left|a_{k}\right|^{1 / k}<2.36
$$

Each $p_{i}$ also has all roots in $[0,1]$. It follows now by Lemma 1 that if $Q_{n}$ is a polynomial of degree $n$ with integer coefficients, and

$$
\left(\left\|Q_{n}\right\|_{[0,1]}\right)^{1 / n} \leq \frac{1}{2.3605}
$$

then $p_{i}$ divides $Q_{n}$.

Also, by Markov's inequality,

$$
\left\|Q_{n}^{(k)}\right\|_{[0,1]} \leq(2 n)^{k}\left\|Q_{n}\right\|_{[0,1]}
$$

and

$$
\begin{aligned}
\left(\left\|Q_{n}^{(k)}\right\|_{[0,1]}\right)^{1 /(n-k)} & \leq(2 n)^{1 /(n-k)}\left(\left\|Q_{n}\right\|_{[0,1]}\right)^{1 /(n-k)} \\
& \leq \frac{1}{2.36}
\end{aligned}
$$

for $n$ sufficiently large compared to $k$. So not only does $p_{i}$ divide $Q_{n}$, but $p_{i}$ divides $Q_{n}^{(k)}$. In particular, each of the factors $p_{i}$ must appear to arbitrarily high multiplicity eventually.

We deduce immediately as above:

Corollary 2.4. The polynomials

$$
p_{0}, p_{1}, \ldots, p_{9}
$$

are the only irreducible polynomials, with all their zeros in $[0,1]$, of the form

$$
p(z)=a_{n} z^{n}+a_{n-1} z^{n-1}+\cdots+a_{0}, \quad a_{i} \in \mathbb{Z}, \quad\left|a_{n}\right|^{1 / n} \leq 2.36 .
$$

Furthermore, the polynomials

$$
\tilde{p}_{0}, \tilde{p}_{1}, \ldots, \tilde{p}_{9},
$$

where $\tilde{p}_{j}(z):=z^{\operatorname{deg}\left(p_{j}\right)} p_{j}(1 / z)$, are the only irreducible polynomials with all their zeros in $[1, \infty)$ that satisfy

$$
N^{1 / d} \leq 2.36
$$

(Here, $N=N\left(p_{j}\right)$ is the norm of $p_{j}$ on $[0,1]$, that is, the product of the zeros of $p_{j}$, and $d=d\left(p_{j}\right)$ is the degree of $\left.p_{j}.\right)$

Proof. This follows easily from the above computations and Lemma 1. (One needs to consider the change of variable $x \rightarrow x(1-x)$.) This should be compared with Smyth [20,21] and Flammang [11], where many similar results are obtained, including forms of the one above. 


\section{Finer StRUCture}

The exact dependence of $\Omega[a, b]$ on the interval $[a, b]$ is interesting and complicated. If we let

$$
\Omega(x):=\Omega[0, x]
$$

then clearly $\Omega$ is a nondecreasing function on $(0, \infty)$. Obviously, $\lim _{x \rightarrow 0} \Omega(x)=0$ (consider $x^{m}$ on $[0, \delta]$ ). So $\Omega(x)$ maps $[0,4]$ onto $[0,1]$. It is an exercise to show that $\Omega$ is in fact continuous. This follows mostly from a theorem of Chebyshev that gives

$$
\left\|p_{n}\right\|_{[0, \delta+\epsilon]} \leq\left(1+k_{\epsilon, \delta}\right)^{n}\left\|p_{n}\right\|_{[0, \delta]}
$$

for every $p_{n} \in \mathcal{P}_{n}$. Here, $k_{\epsilon, \delta}$ is a constant that tends to zero as $\epsilon$ tends to zero, independently of the degree $n$ and independently of $\delta>\delta_{0}>0$.

What is less obvious is that $\Omega(x)$ is locally flat on many intervals. Indeed, it is conceivable that the derivative of $\Omega$ is almost everywhere zero. We cannot prove this. However, we prove the nontrivial fact that $\Omega$ is constant on a specific open neighborhood of 1. See Theorem 3.1 and Corollary 3.12 and compare [1].

We now show that an $n$th integer Chebyshev polynomial on $[0,1]$ has at least $n / 2$ of its zeros at 0 and 1 provided $n$ is large enough. To this end, we need some results on orthogonal Müntz-Legendre polynomials. Let $\Lambda=\left\{\lambda_{i}\right\}_{i=0}^{\infty}$ be a fixed sequence of distinct, nonnegative real numbers. Let

$$
L_{n}(x):=L_{n}\left\{\lambda_{0}, \lambda_{1}, \ldots, \lambda_{n}\right\}(x)=\sum_{j=0}^{n} c_{j, n} x^{\lambda_{j}}, \quad x \in(0, \infty),
$$

with

It is shown in [6] that

$$
c_{j, n}=\frac{\prod_{i=0}^{n-1}\left(\lambda_{j}+\lambda_{i}+1\right)}{\prod_{i=0, i \neq j}^{n}\left(\lambda_{j}-\lambda_{i}\right)} .
$$

$$
\int_{0}^{1} L_{n}(x) L_{m}(x) d x=\frac{\delta_{n, m}}{2 \lambda_{n}+1}, \quad n, m=0,1, \ldots,
$$

where $\delta_{n, m}$ is the Kronecker symbol. From this orthogonality it follows easily that

$$
\frac{1}{\left|c_{n, n}\right| \sqrt{2 \lambda_{n}+1}}=\min \left\{\left\|\sum_{i=0}^{n} a_{i} x^{\lambda_{i}}\right\|_{L_{2}[0,1]}: a_{0}, a_{1}, \ldots, a_{n-1} \in \mathbb{R}, \quad a_{n}=1\right\},
$$

and hence that

for every $a_{0}, a_{1}, \ldots, a_{n} \in \mathbb{R}$.

$$
\left|a_{n}\right| \leq\left|c_{n, n}\right| \sqrt{2 \lambda_{n}+1}\left\|\sum_{i=0}^{n} a_{i} x^{\lambda_{i}}\right\|_{L_{2}[0,1]}
$$

Let $0 \leq k \leq n$ be fixed integers. Let $P_{n} \in \mathcal{P}_{n}$ be of the form

$$
P_{n}(x)=x^{n-k} Q_{k}(x), \quad Q_{k} \in \mathcal{P}_{k}, \quad Q_{k}(0) \neq 0 .
$$

Applying the above inequality with $n$ replaced by $n-k$ and

$$
\lambda_{i}:=n-i, \quad i=0,1, \ldots, n-k,
$$

we obtain that

$$
\begin{aligned}
\left|Q_{k}(0)\right| & \leq \sqrt{2 k+1} \frac{\prod_{i=0}^{n-k-1}(k+(n-i)+1)}{\prod_{i=0}^{n-k-1}((n-i)-k)}\left\|P_{n}\right\|_{L_{2}[0,1]} \\
& =\sqrt{2 k+1}\left(\begin{array}{c}
n+k+1 \\
n-k
\end{array}\right)\left\|P_{n}\right\|_{L_{2}[0,1]}
\end{aligned}
$$


Theorem 3.1. Let $T_{n}:=T_{n}\{[0,1]\} \in \mathcal{Z}_{n}$ be an nth integer Chebyshev polynomial on $[0,1]$. Then $T_{n}$ is of the form

$$
T_{n}(x)=x^{k}(1-x)^{k} S_{n-2 k}(x), \quad S_{n-2 k} \in \mathcal{Z}_{n-2 k},
$$

where $0.26 n<k$ if $n$ is large enough.

As a consequence, there exists an absolute constant $\delta>0$ (independent of $n$ ) so that $T_{n}$ is an nth integer Chebyshev polynomial on larger intervals $[-a, 1+a]$ for every $a \in(0, \delta]$.

Proof. By symmetry, it is sufficient to prove that if

$$
T_{n}(x)=x^{k} Q_{n-k}(x), \quad Q_{n-k} \in \mathcal{Z}_{n-k}, \quad Q_{n-k}(0) \neq 0,
$$

then $0.26 n<k$ if $n$ is large enough.

Observe that $Q_{n-k} \in \mathcal{Z}_{n-k}$ and $Q_{n-k}(0) \neq 0$ implies that $1 \leq\left|Q_{n-k}(0)\right|$. Also, by Proposition 2.2,

$$
\left\|T_{n}\right\|_{L_{2}[0,1]} \leq\left\|T_{n}\right\|_{[0,1]} \leq(2.36+\epsilon)^{-n}
$$

for some $\epsilon>0$, if $n$ is large enough. These, together with (3.1), yield that

$$
1 \leq \sqrt{2 k+1}\left(\begin{array}{c}
n+k+1 \\
n-k
\end{array}\right)(2.36+\epsilon)^{-n}
$$

Hence, by Stirling's formula, if $k / n \leq \alpha<1 / \sqrt{5}$ and $n$ is large enough, then

$$
2.36 \leq \frac{(1+\alpha)^{1+\alpha}}{(2 \alpha)^{2 \alpha}(1-\alpha)^{1-\alpha}}
$$

from which we compute that $\alpha>0.26$. This finishes the proof of the first statement of the theorem. The second statement of the theorem follows from the first one by using a result of Saff and Varga [18] and Chebyshev's inequality. This result of Saff and Varga is formulated in Lemma 3.2.

If $P \in \mathcal{Z}_{k}$ has all its zeros in $[a, b]$, then we say that $P$ is totally real on $[a, b]$. The collection of all totally real polynomials $P \in \mathcal{Z}_{k}$ on $[a, b]$ will be denoted by $\mathrm{TR}_{k}[a, b]$. Let

$$
\operatorname{TR}[a, b]:=\bigcup_{k=0}^{\infty} \operatorname{TR}_{k}[a, b]
$$

Let

$$
P_{0}(x):=x, \quad \text { and } \quad P_{k}(x):=x^{2^{k-1}} P_{k-1}(x-1 / x), \quad k=1,2, \ldots
$$

Then $P_{k} \in \mathrm{TR}_{2^{k}}[\mathbb{R}]$. Let $Q_{k} \in \mathcal{Z}_{2^{k}}$ be defined by

$$
Q_{k}\left(x^{2}\right)=P_{k}(x) P_{k}(-x) .
$$

Then $Q_{k} \in \mathrm{TR}_{2^{k}}[0, \infty]$. Let $R_{k}$ be defined by

$$
R_{k}(x):=x^{2^{k}} Q_{k}(1 / x-1)=: \sum_{j=0}^{2^{k}} a_{j, 2^{k}} x^{j} .
$$


Then $R_{k} \in \mathrm{TR}_{2^{k}}[0,1]$ and

$$
\left|a_{2^{k}, 2^{k}}\right|^{1 / 2^{k}}=\left|F^{[k]}(i)\right|^{1 / 2^{k}} \prod_{j=1}^{k}\left|F^{[j]}(i)\right|^{1 / 2^{j}}, \quad F(x):=x-\frac{1}{x},
$$

where $i$ is the imaginary unit and

$$
F^{[1]}:=F, \quad F^{[j]}:=F^{[j-1]} \circ F, \quad j=2,3, \ldots
$$

It is fairly simple to see that for every fixed $p_{n} \in \mathcal{Z}_{n}$, there exist infinitely many $R_{k}$ with no common factors with $p_{n}$. Since

$$
\gamma:=\lim _{k \rightarrow \infty}\left|F^{[k]}(i)\right|^{1 / 2^{k}} \prod_{j=1}^{k}\left|F^{[j]}(i)\right|^{1 / 2^{j}}=2.37684 \ldots,
$$

the lower bound

$$
\gamma^{-1}=\frac{1}{2.37684 \ldots} \leq \Omega[0,1]
$$

follows from Lemma 1.1 in a canonical fashion. It might be natural to conjecture that $\gamma^{-1}$ is the sharp lower bound. However, using Theorem 3.1, we show that $\gamma^{-1}$ is not the best possible lower bound for $\Omega[0,1]$. For this we need two lemmas. The first one is due to Saff and Varga [18].

Lemma 3.2. Let $\left\{p_{n}\right\}$ be a sequence of polynomials of the form

$$
p_{n}(x)=x^{k(n)} q_{n-k(n)}, \quad q_{n-k(n)} \in \mathcal{P}_{n-k(n)},
$$

where $0 \leq k(n) \leq n$ are integers and where $n \rightarrow \infty$. Suppose that there exists a constant $\theta$ with $0<\theta<1$ so that

$$
\theta \leq \frac{k(n)}{n}, \quad n=1,2, \ldots
$$

If

$$
\limsup _{n \rightarrow \infty}\left\|p_{n}\right\|^{1 / n} \leq 1
$$

then

$$
\lim _{n \rightarrow \infty} p_{n}(x)=0 \quad \text { for all } x \in\left[0, \theta^{2}\right),
$$

uniformly on every closed subset of $\left[0, \theta^{2}\right)$. Moreover, the convergence is geometric, in the sense that for any closed $K \subset\left[0, \theta^{2}\right)$,

$$
\limsup _{n \rightarrow \infty}\left\|p_{n}\right\|_{K}^{1 / n}<1
$$

The proof of the next lemma follows simply from the definition of $R_{k}$ given by (3.2); we omit the details.

Lemma 3.3. For every $\delta \in(0,1)$ there exists a constant $\eta=\eta(\delta)>0$ so that $R_{k}$ has at least $\eta 2^{k}$ zeros in $[0, \delta]$ for every large enough $k$. 
Theorem 3.4. There exists an $\epsilon>0$ so that

$$
(\gamma-\epsilon)^{-1} \leq \Omega[0,1]
$$

where $\gamma=2.37684 \ldots$ is defined by $(3.5)$.

Proof. Let $T_{n}:=T_{n}\{[0,1]\} \in \mathcal{Z}_{n}$ be an $n$th integer Chebyshev polynomial on $[0,1]$. There exists an infinite set $A_{n} \subset \mathbb{N}$ so that $R_{k}$ and $T_{n}$ do not have common factors for every $k \in A_{n}$. By Theorem 3.1 and Lemma 3.2 there exists a constant $\alpha \in(0,1)$ so that

$$
\left|T_{n}(x)\right|^{1 / n} \leq \alpha\left\|T_{n}\right\|_{[0,1]}^{1 / n}, \quad x \in[0,1 / 16],
$$

if $n$ is large enough. In what follows we asume that $n$ is so large that (3.6) holds. By Lemma 3.3 there exists an $\eta>0$ so that $R_{k}$ has at least $\eta 2^{k}$ zeros in $[0,1 / 16]$ if $k$ is large enough. For such a large $k \in A_{n}$, denote the zeros of $R_{k}$ by

$$
(0<) x_{1}<x_{2}<\cdots<x_{2^{k}}(\leq 1)
$$

and let $a_{2^{k}}$ denote the leading coefficient of $R_{k}$. The proof of Lemma 1 shows that

$$
\frac{1}{\left|a_{2^{k}}\right|} \leq \prod_{i=1}^{2^{k}}\left|T_{n}\left(x_{i}\right)\right|^{1 / n}
$$

Observe that

$$
\begin{aligned}
\prod_{i=1}^{2^{k}}\left|T_{n}\left(x_{i}\right)\right|^{1 / n} & =\prod_{\substack{i=1 \\
x_{i} \in[0,1 / 16]}}^{2^{k}}\left|T_{n}\left(x_{i}\right)\right|^{1 / n} \prod_{\substack{i=1 \\
x_{i} \in(1 / 16,1]}}^{2^{k}}\left|T_{n}\left(x_{i}\right)\right|^{1 / n} \\
& \leq \alpha^{\eta 2^{k}}\left(\left\|T_{n}\right\|_{[0,1]}\right)^{2^{k} / n} .
\end{aligned}
$$

Taking the $2^{k}$ th root and combining this with (3.7), we obtain that

$$
\frac{1}{\left|a_{2^{k}}\right|^{1 / 2^{k}}} \leq \alpha^{\eta}\left\|T_{n}\right\|_{[0,1]}^{1 / n} \text {. }
$$

Taking the limit when $k \in A_{n}$ tends to $\infty$, we conclude

$$
\gamma^{-1} \alpha^{-\eta} \leq\left\|T_{n}\right\|_{[0,1]}^{1 / n}, \quad n=0,1, \ldots
$$

Since $0<\alpha<1$ and $0<\eta$, the theorem is proved.

In general, on $[0,1 / m]$ we prove the following lower bound for $\Omega[0,1 / m]$.

Theorem 3.5. We have

$$
\left(m+2-\frac{1}{4(m+1)}\right)^{-1} \leq \Omega[0,1 / m]
$$

for every $m=1,2, \ldots$. 
Proof. Change the definition of $R_{k}$ in $(3.2)$ to

$$
R_{k}(x):=x^{2^{k}} Q_{k}(1 / x-m):=\sum_{j=0}^{2^{k}} a_{j, 2^{k}} x^{j}
$$

Then $R_{k} \in \mathrm{TR}_{2^{k}}[0,1 / m]$ and

$$
\left|a_{2^{k}, 2^{k}}\right|^{1 / 2^{k}}=\left|F^{[k]}(i \sqrt{m})\right|^{1 / 2^{k}} \prod_{j=1}^{k}\left|F^{[j]}(i \sqrt{m})\right|^{1 / 2^{j}}, \quad F(x)=x-\frac{1}{x}
$$

where $i$ is the imaginary unit and $F^{[j]}$ is defined by (3.4). It follows easily again that for every fixed $p_{n} \in \mathcal{Z}_{n}$ there exist infinitely many $R_{k}$ with no common factors with $p_{n}$. Therefore, Lemma 1.1 yields that

$$
\gamma_{m}^{-1} \leq \Omega[0,1 / m]
$$

where

$$
\gamma_{m}:=\lim _{k \rightarrow \infty}\left|F^{[k]}(i \sqrt{m})\right|^{1 / 2^{k}} \prod_{j=1}^{k}\left|F^{[j]}(i \sqrt{m})\right|^{1 / 2^{j}} .
$$

To give an upperbound for $\gamma_{m}$, first note that

$$
\gamma_{m} \leq f(m)\left(\frac{\sqrt{m}+1 / \sqrt{m}+1 /(\sqrt{m}+1 / \sqrt{m})}{\sqrt{m}+2 / \sqrt{m}}\right)^{1 / 4}
$$

where the function $f$ is defined by

$$
f(x):=x \prod_{k=1}^{\infty}\left(1+\frac{k}{x}\right)^{2^{-k}}, \quad x \in(0, \infty) .
$$

To estimate $f(m)$, we distinguish four steps.

Step 1. We have

$$
\limsup _{x \rightarrow \infty}(f(x)-(x+2)) \leq 0
$$

Proof. Indeed,

$$
\begin{aligned}
f(x)-x & \leq x\left(\exp \left(\sum_{k=1}^{\infty} \frac{k 2^{-k}}{x}\right)-1\right) \\
& \leq x\left(\exp \left(\frac{2}{x}\right)-1\right) \underset{x \rightarrow \infty}{\longrightarrow} 2
\end{aligned}
$$


Step 2. The function

$$
g(x):=\log f(x)-\log (x+2)
$$

is nondecreasing on $(0, \infty)$.

Proof. We need to show that

$$
\left(\log x+\left(\sum_{k=1}^{\infty} 2^{-k} \log \left(1+\frac{k}{x}\right)\right)-\log (x+2)\right)^{\prime} \geq 0
$$

on $(0, \infty)$. To this end, it is sufficient to show, in sequence, the following inequalities for all $x \in(0, \infty)$ :

$$
\begin{gathered}
\frac{1}{x}+\sum_{k=1}^{\infty}\left(2^{-k} k \frac{x^{-2}}{1+\frac{k}{x}}\right)-\frac{1}{x+2} \geq 0, \\
\frac{1}{x}-\frac{1}{x+2} \geq \sum_{k=1}^{\infty} 2^{-k} k \frac{1}{x(x+k)}, \\
\frac{1}{x}-\frac{1}{x+2} \geq \sum_{k=1}^{\infty} 2^{-k}\left(\frac{1}{x}-\frac{1}{x+k}\right), \\
\frac{1}{x+2} \leq \sum_{k=1}^{\infty} \frac{2^{-k}}{x+k}, \\
\sum_{k=1}^{\infty} 2^{-k}\left(\frac{1}{x+k}-\frac{1}{x+2}\right) \geq 0, \\
\sum_{k=1}^{\infty} 2^{-k} \frac{2-k}{(x+k)(x+2)} \geq 0 .
\end{gathered}
$$

So

$$
\begin{aligned}
\sum_{k=1}^{\infty} 2^{-k} \frac{2-k}{(x+k)(x+2)} & \geq \sum_{k=1}^{\infty} 2^{-k} \frac{2-k}{(x+1)(x+2)} \\
& =\frac{1}{(x+1)(x+2)}\left(2 \sum_{k=1}^{\infty} 2^{-k}-\sum_{k=1}^{\infty} k 2^{-k}\right) \\
& =\frac{1}{(x+1)(x+2)}(2-2)=0
\end{aligned}
$$

for all $x \in(0, \infty)$, so the statement is proved.

Step 3. By Step 2 the function $h$ defined by

$$
h(x):=\frac{f(x)}{x+2}
$$

is nondecreasing. 
Step 4. We have $f(x) \leq x+2$ for every $x \in(0, \infty)$.

Proof. Otherwise, $f(x) /(x+2)>1+\epsilon$ for some $x$ in $(0, \infty)$ and $\epsilon>0$, hence by Step $3, f(x) /(x+2)>1+\epsilon$ for every large enough $x$, so $f(x)>x+2+2 \epsilon$ for every large enough $x$, which contradicts Step 1.

We conclude that $f(m) \leq m+2$, and the theorem follows from (3.10) and (3.12).

Combining the preceding theorem with a simple computation, we obtain

Theorem 3.6. We have

$$
\left(m+2-\frac{1}{4(m+1)}\right)^{-1} \leq \Omega[0,1 / m]<(m+1.46)^{-1}
$$

if $m \in \mathbb{N}$ is large enough.

Proof. For $\alpha=1.32$,

$$
\left\|x^{\alpha m}(m x-1)((m+1) x-1)\right\|^{1 /(\alpha m+2)} \leq(m+1.46+\epsilon)^{-1}
$$

if $m \in \mathbb{N}$ is large enough and $\epsilon>0$ is small enough.

The next theorem tells us that for $c>8$ and large values of $m \in \mathbb{N}$ the $n$th integer Chebyshev polynomial on $\left[(m+c)^{-1}, m^{-1}\right]$ is forced to have a zero at 0 with large multiplicity. This leads to the interesting observation that if $m \in \mathbb{N}$ and $c>8$ are large enough then the $n$th integer Chebyshev polynomials on $\left[(m+c)^{-1}, m^{-1}\right]$ are exactly the $n$th integer Chebyshev polynomials on $\left[0, m^{-1}\right]$. See Corollary 3.8. It also follows that there exists an absolute constant $c>0$ so that if $n<\mathrm{cm}$, then each of the $n$th integer Chebyshev polynomials $T_{n}:=T_{n}[0,1 / m]$ is of the form $T_{n}(x)=x^{n}$.

Theorem 3.7. Let $m$ be a positive integer and $c>8$. Let

$$
I_{m, c}:=\left[(m+c)^{-1}, m^{-1}\right]
$$

and let $T_{n}:=T_{n}\left\{I_{m, c}\right\} \in \mathcal{Z}_{n}$ be an nth integer Chebyshev polynomial on $I_{m, c}$. Then $T_{n}$ is of the form

$$
T_{n}(x)=x^{k} Q_{n-k}(x), \quad Q_{n-k} \in \mathcal{Z}_{n-k}, \quad Q_{n-k}(0) \neq 0,
$$

with

$$
n\left(1-\frac{8}{\log 2} \frac{1}{m}\right) \leq k
$$

Proof. Chebyshev's inequality $[6, \S 5.1]$ and the explicit form of the (usual noninteger) Chebyshev polynomial of degree $n$ give

$$
|P(y)| \leq \frac{1}{2}\left|\frac{2(y-(a+b) / 2)}{(b-a) / 2}\right|^{n}\|P\|_{[a, b]}, \quad y \notin[a, b],
$$

for every $P \in \mathcal{P}_{n}$. Applying this with

$$
P:=T_{n}, \quad a:=(m+c)^{-1}, \quad b:=m^{-1}, \quad y:=0,
$$


we obtain that

$$
\begin{aligned}
1 \leq\left|Q_{n-k}(0)\right| & \leq 2^{n-k}\left(\frac{2(m+8)}{8}\right)^{n-k}\left\|Q_{n-k}\right\|_{I_{m, 8}} \\
& \leq 2^{n-k}\left(\frac{2(m+8)}{8}\right)^{n-k}\left(\frac{1}{m+8}\right)^{-k}\left\|T_{n}\right\|_{I_{m, 8}} \\
& \leq 2^{n-k}\left(\frac{2(m+8)}{8}\right)^{n-k}\left(\frac{1}{m+8}\right)^{-k} m^{-n} \\
& \leq\left(\frac{1}{2}\right)^{n-k}\left(\frac{m+8}{m}\right)^{n}
\end{aligned}
$$

where we used that

$$
\left\|T_{n}\right\|_{I_{m, 8}} \leq\left\|T_{n}\right\|_{I_{m, c}} \leq\left\|x^{n}\right\|_{I_{m, c}}=m^{-n}
$$

and the observation that $Q_{n-k} \in \mathcal{Z}_{k}$ and $Q_{n-k}(0) \neq 0$ implies that $1 \leq\left|Q_{n-k}(0)\right|$. We conclude that

$$
(n-k) \log 2 \leq n \log \left(1+8 m^{-1}\right) \leq n\left(8 m^{-1}\right),
$$

and the theorem follows.

Corollary 3.8. Suppose $m \in \mathbb{N}, m \geq 32 / \log 2$, and $c \geq 32 / \log 2$. Let

$$
T_{n}:=T_{n}\left\{\left[(m+c)^{-1}, m^{-1}\right]\right\} \in \mathcal{Z}_{n}
$$

be an $n$th integer Chebyshev polynomial on $\left[(m+c)^{-1}, m^{-1}\right]$. Then $T_{n}$ is also an $n$th integer Chebyshev polynomial on $[0,1 / \mathrm{m}]$.

Proof. Combine Theorem 3.7 and Lemma 3.2.

The next theorem shows that if $a \in(0,1 / 8)$ then an $n$th integer Chebyshev polynomial on $[a, 1]$ has at least $(0.17) n$ zeros at 0 . See Corollary 3.10 . It follows easily from this that the $n$th integer Chebyshev polynomials on $\left[0,1-(0.17)^{2}\right]$ are exactly the $n$th integer Chebyshev polynomials on [0,1]. See Corollary 3.12. This implies that the function $\Omega(x):=\Omega[0, x]$ is constant on $\left[1-(0.17)^{2}, 1\right]$. This, together with the second statement of Theorem 3.1, yields that $\Omega$ is constant on an open neighborhood of 1 .

Theorem 3.9. Let $a \in(0,1)$. Let $T_{n}:=T_{n}\{[a, 1]\} \in \mathcal{Z}_{n}$ be an nth integer Chebyshev polynomial on $[a, 1]$. Suppose that $\alpha \in(0,1 / \sqrt{5}]$ satisfies

$$
2.36 \geq \frac{(1+\alpha)^{1+\alpha}}{(2 \alpha)^{2 \alpha}(1-\alpha)^{1-\alpha}}\left(1+\frac{2 a}{1-a}+\sqrt{\left(1+\frac{2 a}{1-a}\right)^{2}-1}\right)^{\alpha}
$$

Then

$$
T_{n}(x)=x^{k} Q_{n-k}(x), \quad Q_{n-k} \in \mathcal{Z}_{n-k}, \quad Q_{n-k}(0) \neq 0,
$$

with $\alpha n \leq k$ for every sufficiently large $n$. 
Proof. Combining inequality (3.1), Chebyshev's inequality [6, §5.1], the explicit form of the (usual noninteger) Chebyshev polynomial of degree $n$, and Proposition 2.2 , we obtain that

$$
\begin{aligned}
1 & \leq\left|Q_{k}(0)\right| \leq \sqrt{2 k+1}\left(\begin{array}{c}
n+k+1 \\
n-k
\end{array}\right)\left\|T_{n}\right\|_{L_{2}[0,1]} \\
& \leq \sqrt{2 k+1}\left(\begin{array}{c}
n+k+1 \\
n-k
\end{array}\right)\left(1+\frac{2 a}{1-a}+\sqrt{\left(1+\frac{2 a}{1-a}\right)^{2}-1}\right)^{k}\left\|T_{n}\right\|_{[0, a]} \\
& \leq \sqrt{2 k+1}\left(\begin{array}{c}
n+k+1 \\
n-k
\end{array}\right)\left(1+\frac{2 a}{1-a}+\sqrt{\left(1+\frac{2 a}{1-a}\right)^{2}-1}\right)^{k}(2.36+\epsilon)^{-n}
\end{aligned}
$$

for some $\epsilon>0$. Hence by Stirling's formula, if $k / n \leq \alpha \leq 1 / \sqrt{5}$ and $n$ is large enough, then

$$
2.36<\frac{(1+\alpha)^{1+\alpha}}{(2 \alpha)^{2 \alpha}(1-\alpha)^{1-\alpha}}\left(1+\frac{2 a}{1-a}+\sqrt{\left(1+\frac{2 a}{1-a}\right)^{2}-1}\right)^{\alpha}
$$

This contradicts the assumption of the theorem.

Corollary 3.10. Let $a \in(0,1 / 8)$. Let $T_{n}:=T_{n}\{[a, 1]\} \in \mathcal{Z}_{n}$ be an nth integer Chebyshev polynomial on $[0, a]$. Then

$$
T_{n}(x)=x^{k} Q_{n-k}(x), \quad Q_{n-k} \in \mathcal{Z}_{n-k}, \quad Q_{n-k}(0) \neq 0,
$$

with $0.17 n \leq k$ for every sufficiently large $n$.

Proof. This follows from Corollary 3.9.

Corollary 3.11. Let $a \in\left(0,(0.17)^{2}\right]$. Let $T_{n}:=T_{n}\{[a, 1]\} \in \mathcal{Z}_{n}$ be an $n$th integer Chebyshev polynomial on $[a, 1]$. Then $T_{n}$ is an nth integer Chebyshev polynomial on $[0,1]$ for every sufficiently large $n$.

Proof. Combine Corollary 3.10 and Lemma 3.2.

Corollary 3.12. Let $a \in\left(0,(0.17)^{2}\right]$. Let $T_{n}:=T_{n}\{[0,1-a]\} \in \mathcal{Z}_{n}$ be an nth integer Chebyshev polynomial on $[0,1-a]$. Then $T_{n}$ is an nth integer Chebyshev polynomial on $[0,1]$ for every sufficiently large $n$.

Proof. Apply Corollary 3.11 with the substitution $y=1-x$.

To find the value $\lim \sup _{m}\left((\Omega[0,1 / m])^{-1}-m\right)$ seems to be an interesting problem. Theorem 3.6 yields that this value is at most 2 , and it may be suspected that it is exactly 2. However, the next corollary shows that it is less than 2. (This should be compared with Amoroso [1] where a weaker result is proved with $2-\epsilon$ replaced by 2.)

Corollary 3.13. Let

$$
\gamma_{m}:=(\Omega[0,1 / m])^{-1}-m
$$


Then there exists an $\epsilon>0$ so that

$$
\limsup _{m} \gamma_{m} \leq 2-\epsilon
$$

Proof. This follows from Theorem 3.5, Corollary 3.8 and Lemmas 3.1 and 3.3. The details are similar to those given in the proof of Theorem 3.4, so we omit them.

\section{The Schur-Siegel trace Problem}

Let $\alpha:=\alpha_{1}$ be an algebraic integer with conjugates $\alpha_{2}, \ldots, \alpha_{n}$. We say that $\alpha$ is totally real (positive) if all the $\alpha_{i}$ are real (positive). The trace of a totally positive algebraic integer is the sum $\alpha_{1}+\alpha_{2}+\cdots+\alpha_{n}$, which is just, up to the sign, the second-highest coefficient of the minimal polynomial. Except for finitely many explicit exceptions, if $\alpha$ is a totally real algebraic integer of degree $d>1$, then

$$
\begin{aligned}
& \frac{\alpha_{1}+\alpha_{2}+\cdots+\alpha_{d}}{d} \geq 1.648, \quad \text { Schur }(1918) \\
& \frac{\alpha_{1}+\alpha_{2}+\cdots+\alpha_{d}}{d} \geq 1.733, \quad \text { Siegel }(1945) \\
& \frac{\alpha_{1}+\alpha_{2}+\cdots+\alpha_{d}}{d} \geq 1.771, \quad \text { Smyth (1983). }
\end{aligned}
$$

See $[16,19,20,21]$. Note that $4 \cos ^{2}(\pi / p)$ is a totally positive algebraic integer of degree $(p-1) / 2$ and trace $p-2$ for $p$ prime. So the best constant in the above theorem is less than 2. The connection with the integer Chebyshev Problem is the content of the next proposition.

Proposition 4.1. Suppose $m$ is a positive integer and

$$
\Omega[0,1 / m]<(m+\delta)^{-1} .
$$

Then, with at most finitely many exceptions,

$$
\frac{\alpha_{1}+\alpha_{2}+\cdots+\alpha_{d}}{d} \geq \delta
$$

for every totally positive algebraic integer $\alpha_{1}$ of degree $d>1$ with conjugates $\alpha_{2}, \ldots, \alpha_{d}$.

Proof. Suppose $p$ is the minimal polynomial for $\alpha$ and

$$
p(x):=x^{d}-a_{d-1} x^{d-1}+\cdots+a_{0} ;
$$

then $\alpha_{1}+m, \alpha_{2}+m, \ldots, \alpha_{d}+m$ are conjugate roots of $q \in \mathcal{Z}_{d}$ defined by

$$
q(x)=x^{d}-\left(a_{d-1}+m d\right) x^{d-1}+\cdots+b_{0} .
$$

Now by the arithmetic-geometric mean inequality

$$
\begin{aligned}
b_{0}^{1 / d} & =\left(\left(\alpha_{1}+m\right)\left(\alpha_{1}+m\right) \cdots\left(\alpha_{d}+m\right)\right)^{1 / d} \\
& \leq \frac{\alpha_{1}+\alpha_{2}+\cdots+\alpha_{d}+d m}{d}=\frac{a_{d-1}}{d}+m
\end{aligned}
$$


We apply Lemma 1.1 to

$$
\tilde{q}(x):=x^{d} q(1 / x),
$$

which has all its roots in $(0,1 / m)$ and is irreducible, to conclude that either

$$
\frac{a_{d-1}}{d}+m>m+\delta
$$

(which is the conclusion we want) or $\tilde{q}(x)$ is a factor of all $n$th integer Chebyshev polynomials on $[0,1 / m]$, provided $n$ is large enough.

Corollary 4.2. If $\alpha_{1}$ is a totally positive algebraic integer of degree $d>1$ with conjugates $\alpha_{2}, \ldots, \alpha_{d}$, then

$$
\frac{\alpha_{1}+\alpha_{2}+\cdots+\alpha_{d}}{d}>1.752
$$

with at most finitely many exceptions. (There are no exceptions of degree greater than 8.)

This is not as good as Smyth's result. It, however, follows immediately from a computation, as in $\S 2$, which shows that

$$
\Omega[0,1 / 200]<\frac{1}{201.752},
$$

and gives the factors of an example which yields the above upper bound.

\section{OPEN PROBLEMS}

There are a myriad of open problems in and around integer Chebyshev polynomials. We formulate a few of them as questions.

Q1. Find a reasonable algorithm for exactly computing integer Chebyshev polynomials on $[0,1]$ that would work up to, say, degree 200.

Q2. Are the integer Chebyshev polynomials eventually unique?

Q3. Do the integer Chebyshev polynomials on $[0,1]$ have all their zeros in $[0,1]$ ?

Q4. Determine $\Omega[0, \alpha]$ exactly for any $0<\alpha<4$.

Q5. Determine the limit (or the limsup, if the limit does not exist) of

$$
(\Omega[0,1 / m])^{-1}-m \text {. }
$$

Q6. Are all the irreducible factors of the integer Chebyshev polynomials on $[0,1]$ forced to be factors as in Lemma 1.1? That is, are all irreducible factors $q$ of the form

$$
q(x)=a_{k} x^{k}+a_{k-1} x^{k-1}+\cdots+a_{0},
$$

with all their zeros in $[0,1]$, and with

$$
\left|a_{k}\right|^{1 / k}<(\Omega[0,1])^{-1} ?
$$

Q7. Show that there exist infinitely many irreducible polynomials with integer coefficients which divide an $n$th integer Chebyshev polynomial on $[0,1]$ for some $n$.

Added in proof. In a preprint entitled "On integer Chebyshev polynomials" Habsieger and Salvy compute an $n$th integer Chebyshev polynomial on $[0,1]$ for every $n=1,2, \ldots, 75$. The 70 th such polynomial has a factor with four nonreal zeros. This gives partial answers to Q3 and Q6 above. 


\section{APPENDIX}

The following is a list of all polynomials of degree at most $n$, for $n=1,2, \ldots, 22$, which minimize $\left\|p_{n}\right\|_{L_{2}[0,1]}$ subject to $p_{n} \in \mathcal{Z}_{n}$. This computation was done in pari by using the minum function.

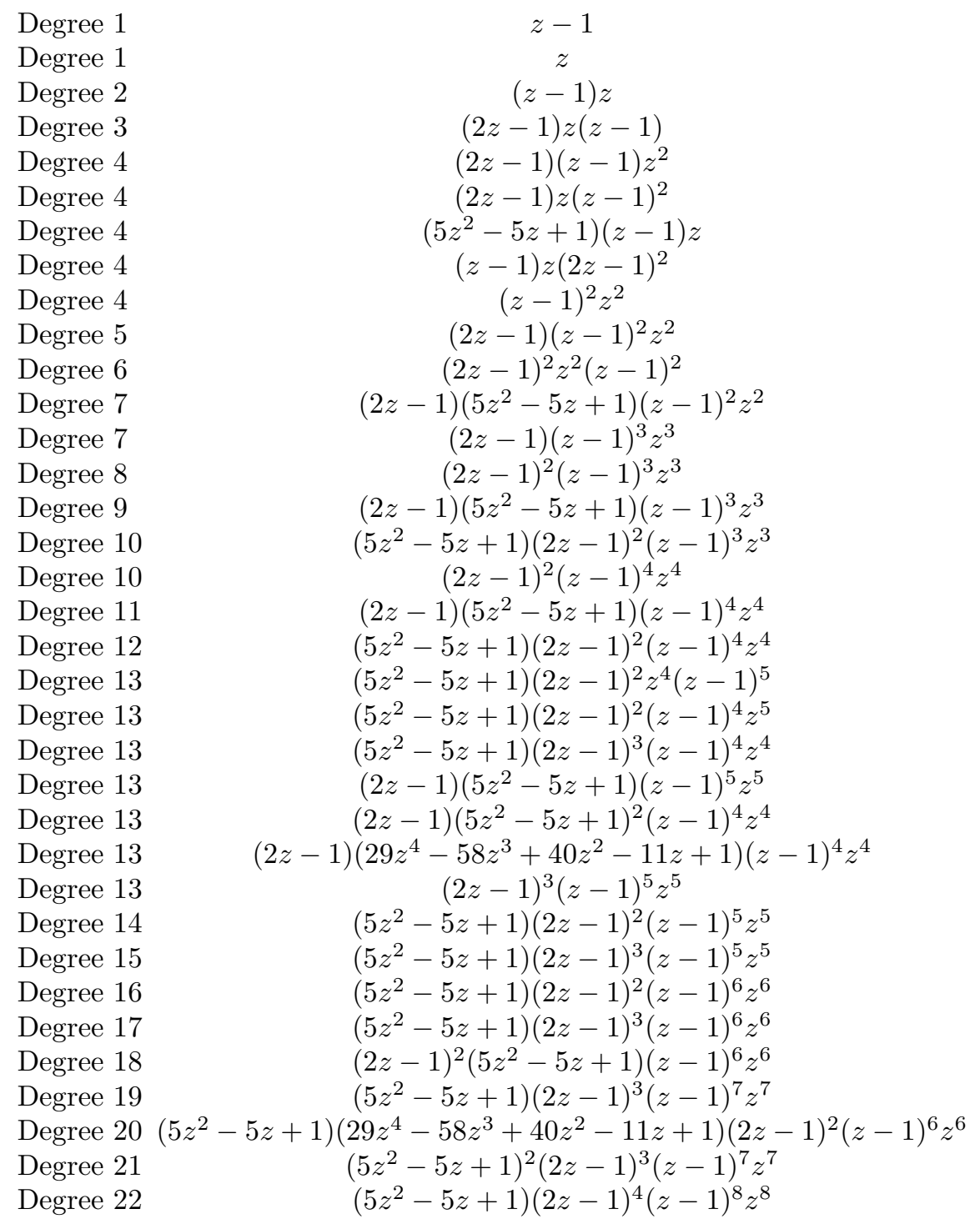

\section{REFERENCES}

1. F. Amoroso, Sur le diamètre transfini entier d'un intervalle réel, Ann. Inst. Fourier, Grenoble 40 (1990), 885-911. MR 92j:11070

2. E. Aparicio, Methods for the approximate calculation of minimum uniform Diophantine deviation from zero on a segment, Rev. Mat. Hisp.-Amer. 38 (1978), 259-270 (Spanish). MR 80i:10049 
3. N New bounds on the minimal Diophantine deviation from zero on $[0,1]$ and $[0,1 / 4]$, Actus Sextas Jour. Mat. Hisp.-Lusitanas (1979), 289-291.

4. _ On some systems of algebraic integers of D.S. Gorshkov and their application in calculus, Rev. Mat. Hisp.-Amer. 41 (1981), 3-17 (Spanish).

5. On some results in the problem of Diophantine approximation of functions by polynomials, Proc. Steklov Inst. Math. 163 (1985), 7-10. MR 86d:41001

6. P. Borwein and T. Erdélyi, Polynomials and polynomial inequalities, Springer-Verlag, New York, 1995.

7. P. Borwein and C. Ingalls, The Prouhet Tarry Escott problem revisited, Math. Enseign. 40 (1994), 3-27.

8. G. V. Chudnovsky, Number theoretic applications of polynomials with rational coefficients defined by extremality conditions, Arithmetic and Geometry, Vol. I (M. Artin and J. Tate, eds.), Progress in Math., Vol. 35, Birkhäuser, Boston, 1983, pp. 61-105. MR 86c:11052

9. M. Fekete, Über die Verteilung der Wurzeln bei gewissen algebraischen Gleichungen mit ganzzahligen Koeffizienten, Math. Zeit. 17 (1923), 228-249.

10. Le Baron O. Ferguson, Approximation by polynomials with integral coefficients, Math. Surveys monographs, Vol. 17, Amer. Math. Soc., Providence, R. I., 1980. MR 81g:41011

11. V. Flammang, Sur la longeur des entiers algébriques totalement positifs, preprint.

12. D. Hilbert, Ein Beitrag zur Theorie des Legendreschen Polynoms, Acta Math. 18 (1894), $155-159$.

13. B. Kashin, On algebraic polynomials with integer coefficients with least deviation from zero on an interval, preprint.

14. A. K. Lenstra, H. W. Lenstra Jr., and L. Lovász, Factoring polynomials with rational coefficients, Math. Ann. 261 (1982), 515-534. MR 94a:12002

15. H. L. Montgomery, Ten Lectures on the Interface Between Analytic Number Theory and Harmonic Analysis, CBMS, Vol. 84, Amer. Math. Soc., Providence, R. I., 1994. CMP 95:02

16. I. Schur, Über die Verteilung der Wurzeln bei gewissen algebraischen Gleichungen mit ganzzahligen Koeffizienten, Math. Zeit. 1 (1918), 377-402.

17. G. Pólya and G. Szegö, Problems and Theorems in Analysis, Volume I, Springer-Verlag, New York, 1972. MR 49:8782

18. E. B. Saff and R. S. Varga, On lacunary incomplete polynomials, Math. Z. 177 (1981), 297314. MR 83a: 41008

19. C. L. Siegel, The trace of totally positive and real algebraic integers, Annals of Math. 46 (1945), 302-314. MR 6:257a

20. C. J. Smyth, The mean values of totally real algebraic integers, Math. Comp. 42 (1984), 663-681. MR 86e: 11115

21. MR 86f: 11091

Department of Mathematics and Statistics, Simon Fraser University, Burnaby, B.C., CANADA V5A 1S6

E-mail address: pborwein@cecm.sfu.ca

Department of Mathematics, Texas A \& M University, College Station, Texas 77843

E-mail address: terdelyi@math.tamu.edu 